\title{
Structural Perturbation of Superfolder GFP in the Presence of Guanidine Thiocyanate
}

\author{
Olesya V. Stepanenko, ${ }^{1}$ Olga V. Stepanenko, ${ }^{1}$ Irina M. Kuznetsova, ${ }^{1}$ \\ Vladyslav V. Verkhusha, ${ }^{2}$ and Konstantin K. Turoverov ${ }^{1}$ \\ ${ }^{1}$ Laboratory of Structural Dynamics, Stability and Folding of Proteins, Institute of Cytology Russian \\ Academy of Sciences, Tikhoretsky Avenue 4, Saint-Petersburg 194064, Russia \\ ${ }^{2}$ Department of Anatomy and Structural Biology, Albert Einstein College of Medicine, 1300 Morris Park \\ Avenue, Bronx, NY 10461, USA
}

Correspondence should be addressed to Olesya V. Stepanenko, lvs@incras.ru

Copyright (C) 2012 Olesya V. Stepanenko et al. This is an open access article distributed under the Creative Commons Attribution License, which permits unrestricted use, distribution, and reproduction in any medium, provided the original work is properly cited.

\begin{abstract}
The guanidine-thiocyanate-induced denaturation-renaturation of sfGFP was studied. It was shown that the disruption of sfGFP native structure occurs in the range of guanidine thiocyanate concentrations from 0.5 to $2.5 \mathrm{M}$. This process was accompanied by simultaneous changes of all recorded parameters. It was found that the small guanidine thiocyanate concentrations (less then 0.1-0.2 M) triggered local structural disturbances in protein which result in significant decrease of chromophore and tryptophan fluorescence intensity and change of protein visible absorption spectrum.
\end{abstract}

Keywords: Fluorescent proteins, super-folder GFP, folding of proteins with beta-barrel topology, small guanidine thiocyanate concentrations

\section{Introduction}

A broad variety of fluorescent proteins (FPs) and their engineered analogs are currently used as fluorescent tags of different color's in bioimaging [1-5]. The application of FPs has facilitated the routine monitoring of gene activation and the selective labeling of single proteins, cellular organelles, or the whole cells $[6,7]$. A number of FP-based biosensors have been designed to sense the properties of the cell's environment such as $\mathrm{pH}$, ion flux, redox potential [8-12]. Genetically encoded FP-containing biosensors based on fluorescence resonance energy transfer (FRET) have been widely adopted for the investigation of protein-protein interactions and other biologically relevant events occurring in the living cell $[1,13]$. FPs with light-modulated spectral properties, collectively termed photoactivatable fluorescent proteins (PAFPs), allow to break diffraction limit and obtain the superresolution imaging in fixed and live cells [3]. Recent development of FPs emitting in far-red region and FPs possessing large Stokes shifts have enabled visualization of cellular processes in living animals $[14,15]$.

The key feature of all FPs which attracts an enormous interest to them is ability to self-generate the intrinsic chromophore from three amino acids in the position 65-67 without engaging any cofactors or enzymatic components [16]. Chromophore is encapsulated in the rigid $\beta$-barrel-like shell 
of protein matrix which functions are to protect the chromophore from the environment and restrict its flexibility thus preventing from nonradiative deactivation. Moreover, correct protein folding results in the proper orientation of the catalytic amino acids near the chromophore-forming tripeptide and induces chromophore maturation. Thus, the study of FP's folding and structural dynamics is of high interest.

FPs are prone to aggregate leading to the irreversibility of denaturation and impeding the investigation of FPs folding-unfolding. Superfolder, or sfGFP, is the only FP free of this drawback [17]. In this work we studied the denaturant-induced structural changes of sfGFP using spectroscopic techniques (absorbance, fluorescence, circular dichroism) and size-exclusion chromatography.

\section{Materials and Methods}

The plasmid pET-28a (+)-sfGFP encoding superfolder GFP [17] with polyhistidine tags was constructed as described previously [18] and was transformed into an Escherichia coli BL21(DE3) host (Invitrogen). The sfGFP expression was induced by an incubation of the cells with $0.5 \mathrm{mM}$ IPTG (Fluka, Switzerland) during $24 \mathrm{~h}$ at $25^{\circ} \mathrm{C}$, and protein was purified with Ni-NTA agarose (GE Healthcare, Sweden). The purity of the recombinant proteins was not less than 95\%, as indicated by SDS-PAGE. Guanidine thiocyanate (Fluka, Switzerland) was used without further purification. Measurements were performed in $50 \mathrm{mM}$ TrisCl buffer, $\mathrm{pH}$ 8.0. Protein concentration was $0.15 \mathrm{mg} / \mathrm{mL}$.

Absorption spectra were recorded using an EPS-3T (Hitachi, Japan) spectrophotometer. The experiments were performed in microcells 101.016-QS $5 \mathrm{~mm} \times 5 \mathrm{~mm}$ (Hellma, Germany) at room temperature.

Fluorescence experiments were carried out using a Cary Eclipse spectrofluorimeter (Varian, Australia) and a homemade spectrofluorimeter for registration of fluorescence polarization [19]. Protein intrinsic fluorescence was excited at the long-wave absorption spectrum edge $(297 \mathrm{~nm})$, where the contribution of tyrosine residues in the bulk protein fluorescence is negligible. Specific green fluorescence of sfGFP was excited at 365 and $470 \mathrm{~nm}$, and emission was detected at $510 \mathrm{~nm}$. The position and form of the fluorescence spectra were characterized by the parameter $A=I_{320} / I_{365}$, where $I_{320}$ and $I_{365}$ are fluorescence intensities at $\lambda_{\mathrm{em}}=320$ and $365 \mathrm{~nm}$, respectively [20]. The values of parameter $A$ and of fluorescence spectrum were corrected by the instrument sensitivity. The anisotropy of tryptophan fluorescence was calculated by $r=\left(I_{V}^{V}-G I_{H}^{V}\right) /\left(I_{V}^{V}+2 G I_{H}^{V}\right)$, where $I_{V}^{V}$ and $I_{H}^{V}$ are the vertical and horizontal components of fluorescence intensity excited by vertically polarized light and $G$ is the relation of vertical and horizontal components of fluorescence intensity excited by horizontally polarized light $\left(G=I_{V}^{H} / I_{H}^{H}\right), \lambda_{\mathrm{em}}=365 \mathrm{~nm}$ [19]. All kinetic experiments were performed in microcells FLR $10 \mathrm{~mm} \times 10 \mathrm{~mm}$ (Varian, Australia). Unfolding of the protein was initiated by manual mixing of protein solution $(50 \mathrm{mkl})$ with buffer containing desired GTC concentrations $(450 \mathrm{mkl})$. The dead time was determined from the control experiments to be about $4 \mathrm{~s}$ [20]. The dependences of different fluorescent characteristics of sfGFP on GTC concentration were recorded after protein incubation in the solution of appropriate concentration at $23^{\circ} \mathrm{C}$ during $2,24,45,69$, and $95 \mathrm{~h}$. The spectrofluorimeter was equipped with thermostat that held a constant temperature of $23^{\circ} \mathrm{C}$.

The gel filtration experiments of sfGFP unfolded in GTC were performed on a Superdex75 PC 3.2/30 column (GE Healthcare, Sweden) using an AKTApurifier system (GE Healthcare, Sweden). Solutions of sfGFP were preincubated at $23^{\circ} \mathrm{C}$ for $24 \mathrm{~h}$ in the presence of the desired GTC concentration. Then $10 \mu \mathrm{L}$ of this solution was loaded on a column equilibrated with the same GTC concentration. The 
change of hydrodynamic dimensions of sfGFP was evaluated as a change of elution volume of sfGFP. In the transition region the averaged elution volume of sfGFP was calculated by $V=f_{c} V_{c}+f_{d} V_{d}$, where $V_{c}$ and $V_{d}$ are the elution peaks of compact and denatured molecules, and $f_{c}$ and $f_{d}$ are the portion of compact and denatured molecules estimated as area of the corresponding peak.

\section{Results and Discussion}

Figure 1 shows denaturation curves of different parameters of tryptophan fluorescence (fluorescence intensity, parameter $A$, and fluorescence anisotropy; excitation at $297 \mathrm{~nm}$ ) and sfGFP chromophore fluorescence intensity excited at two wavelength (365 and $470 \mathrm{~nm}$; registration at $510 \mathrm{~nm}$ ) as a function of final GTC concentration recorded after 1 to $94 \mathrm{~h}$ of equilibration of the protein in the presence of desired denaturant concentration. It was shown that GTC-induced unfolding of sfGFP reaches quasiequilibrium after 24 hours in contrast to several days in the case of GdnHCl-induced denaturation [21, 22]. Further incubation of sfGFP in the presence of GTC, at least up to 4 days, does not result in measurable changes of recorded characteristics. Apparently, the unfolding of protein structure takes place in the range of GTC concentrations from 0.5 to $2.5 \mathrm{M}$. This process was accompanied by simultaneous changes of all recorded parameters. In the subrange of small GTC concentrations (less then $0.1-0.2 \mathrm{M}$ ) we have observed no noticeable changes of tertiary protein structure as indicated by fluorescence anisotropy and parameter $A$ (Figures 1(c) and 1(d)). Nevertheless gel filtration experiments showed a slight increase of hydrodynamic dimensions of sfGFP (Figure 1(j)). At the same time small amount of GTC induces significant decrease of chromophore (Figures 1(e) and 1(f)) and tryptophan fluorescence intensity (Figures 1(a) and 1(b)) and change of protein visible absorption spectrum (Figure 1(i)). Visible absorption spectra of sfGFP demonstrated pronounced drop of absorption band corresponding to the anionic form of chromophore with concomitant rise of absorption band corresponding to neutral chromophore. These data indicate that the small addition of GTC changes the balance between sfGFP molecules bearing neutral and anionic chromophores. Denaturation curves of chromophore fluorescence intensity were corrected to take into account the change of absorption of anionic and neutral chromophore in the presence of GTC (Figures 1(g) and 1(h)). The response of thus corrected chromophore fluorescence excited at two wavelengths is completely different. Excitation at $365 \mathrm{~nm}$ results in significant fluorescence drop (up to $13 \%$ of native protein signal), while excitation at $470 \mathrm{~nm}$ leads to a $20 \%$ increase of fluorescence with respect to native sfGFP. Significant decrease of fluorescence quantum yield under excitation at absorption band of protonated chromophore could be concerned with inhibition of proton transfer and thus chromophore ionization in the presence of small GTC concentrations. We suppose that all observed changes induced by predenaturing GTC concentrations are a result of local structural perturbations of sfGFP rather than global reorganization of protein structure.

Renaturation of sfGFP was induced by dilution of predenatured protein in $2.2 \mathrm{M}$ GTC to the various final denaturant concentrations (Figure 1). Refolding reaches equilibrium after 24 hours of protein incubation. It was shown that under strongly refolding conditions (final concentration of GTC is $0.22 \mathrm{M}$ ) the recovery of all recorded characteristics of sfGFP takes place indicating the reversibility of protein unfolding. The unfolding and refolding curves in the transition area do not coincide showing apparent hysteresis. Hysteretic behavior observed during folding of sfGFP is supposed to be connected with the presence of the chromophore inside the barrel which has to be locked in the correct active form 


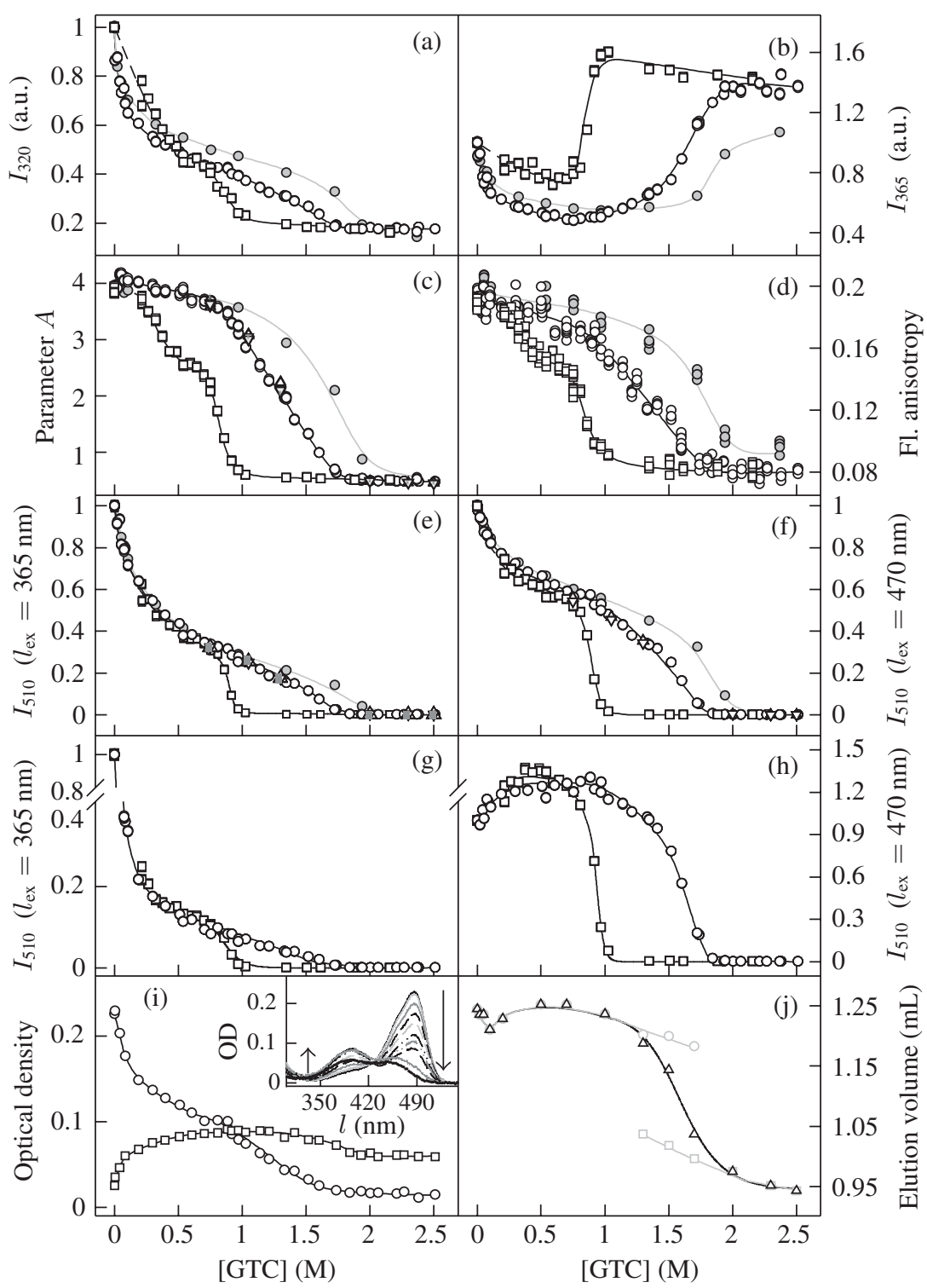

Figure 1: GTC-induced folding-unfolding of sfGFP recorded by the changes of fluorescence intensity at $320 \mathrm{~nm}$ (a) and at $365 \mathrm{~nm}$ (b), parameter $A$ (c), fluorescence anisotropy at $365 \mathrm{~nm}$ (d), and chromophore fluorescence intensity excited at $365 \mathrm{~nm}$ (e) and $470 \mathrm{~nm}$ (f). Unfolding and refolding are presented by circles and squares, respectively. Measurements were performed after $1 \mathrm{~h}$ (closed circles) and $24 \mathrm{~h}$ (open circles and squares), $45 \mathrm{~h}$ (triangles), $69 \mathrm{~h}$ (reversed triangles), and $94 \mathrm{~h}$ (gray crosses) incubation of native or denatured protein in the presence of corresponding denaturant concentration. Data presented in (e) and (f) were corrected to take into account the change of optical density at the wavelength of excitation ( $\mathrm{g}$ and $\mathrm{h}$ ). The conformational changes of sfGFP are further characterized by the changes of absorbance at 390 (squares, i) and $490 \mathrm{~nm}$ (circles, i). Insert to panel (i): The visible fluorescence spectra of sfGFP in the presence of increasing GTC concentration (shown by arrows). The change of sfGFP hydrodynamic dimensions during GTC-induced denaturation was studied by gel filtration method (j). The position of elution peaks of compact and denatured molecules (gray circles and squares) and the change of averaged elution volume of sfGFP (black triangles) are shown. 
in the protein core on the latest folding step [23]. Indeed the mutant variants of green FP which unable to form chromophore show no hysteresis of the unfolding and refolding curves [23].

The refolding curves of parameter $A$ and fluorescence anisotropy for sfGFP exhibit the presence of plateau in the range of GTC concentration from 0.5 to $0.8 \mathrm{M}$ (Figures 1(c) and 1(d)), while fluorescence intensity of sfGFP chromophore measured during denaturation and renaturation experiments coincide in this range of GTC concentrations (Figures 1(e) and 1(f)). In the recent works by molecular dynamics simulations it was shown that initial fast formation of sfGFP $\beta$-barrel is followed by a slow search through chromophore isomerization and structural fluctuations toward the locked active native fold [24]. During second folding step, the final strands incorporating into the barrel and structural rearrangement at the lid of the barrel there occur $[24,25]$. It should be pointed out that Trp 57 is near to those $\beta$-strads. Probably sfGFP refolding is accompanied by accumulation of intermediate state in which a core around chromophore is organized while structure around Trp 57 is not formed.

\section{Acknowledgments}

This work was in part supported by the Ministry of Education and Science (Contracts 02.740.11.5141), Program MCB RAS, and SPb government (to O.V. Steparenko).

\section{References}

[1] Y. Wang, J. Y. J. Shyy, and S. Chien, "Fluorescence proteins, live-cell imaging, and mechanobiology: seeing is believing," Annual Review of Biomedical Engineering, vol. 10, pp. 1-38, 2008.

[2] A. A. Pakhomov and V. I. Martynov, "GFP family: structural insights into spectral tuning," Chemistry and Biology, vol. 15, no. 8, pp. 755-764, 2008.

[3] B. Wu, K. D. Piatkevich, T. Lionnet, R. H. Singer, and V. V. Verkhusha, "Modern fluorescent proteins and imaging technologies to study gene expression, nuclear localization, and dynamics," Current Opinion in Cell Biology, vol. 23, no. 3, pp. 310-317, 2011.

[4] W. B. Frommer, M. W. Davidson, and R. E. Campbell, "Genetically encoded biosensors based on engineered fluorescent proteins," Chemical Society Reviews, vol. 38, no. 10, pp. 2833-2841, 2009.

[5] O. V. Stepanenko, V. V. Verkhusha, I. M. Kuznetsova, V. N. Uversky, and K. K. Turoverov, "Fluorescent proteins as biomarkers and biosensors: throwing color lights on molecular and cellular processes," Current Protein and Peptide Science, vol. 9, no. 4, pp. 338-369, 2008.

[6] D. M. Chudakov, M. V. Matz, S. Lukyanov, and K. A. Lukyanov, "Fluorescent proteins and their applications in imaging living cells and tissues," Physiological Reviews, vol. 90, no. 3, pp. 11031163, 2010.

[7] R. N. Day and M. W. Davidson, "The fluorescent protein palette: tools for cellular imaging," Chemical Society Reviews, vol. 38, no. 10, pp. 2887-2921, 2009.

[8] G. S. Baird, D. A. Zacharias, and R. Y. Tsien, "Circular permutation and receptor insertion within green fluorescent proteins," Proceedings of the National Academy of Sciences of the United States of America, vol. 96, no. 20, pp. 11241-11246, 1999.

[9] R. Bizzarri, M. Serresi, S. Luin, and F. Beltram, "Green fluorescent protein based pH indicators for in vivo use: a review," Analytical and Bioanalytical Chemistry, vol. 393, no. 4, pp. 1107-1122, 2009. 
[10] G. T. Hanson, R. Aggeler, D. Oglesbee et al., "Investigating mitochondrial redox potential with redox-sensitive green fluorescent protein indicators," The Journal of Biological Chemistry, vol. 279, no. 13, pp. 13044-13053, 2004.

[11] T. Mizuno, K. Murao, Y. Tanabe, M. Oda, and T. Tanaka, "Metal-ion-dependent GFP emission in vivo by combining a circularly permutated green fluorescent protein with an engineered metal-ionbinding coiled-coil," Journal of the American Chemical Society, vol. 129, no. 37, pp. 11378-11383, 2007.

[12] E. A. Souslova, V. V. Belousov, J. G. Lock et al., "Single fluorescent protein-based Ca2+ sensors with increased dynamic range," BMC Biotechnology, vol. 7, article 37, 2007.

[13] A. Ibraheem and R. E. Campbell, "Designs and applications of fluorescent protein-based biosensors," Current Opinion in Chemical Biology, vol. 14, no. 1, pp. 30-36, 2010.

[14] D. Shcherbo, I. I. Shemiakina, A. V. Ryabova et al., "Near-infrared fluorescent proteins," Nature Methods, vol. 7, no. 10, pp. 827-829, 2010.

[15] K. D. Piatkevich, J. Hulit, O. M. Subach et al., "Monomeric red fluorescent proteins with a large Stokes shift," Proceedings of the National Academy of Sciences of the United States of America, vol. 107, no. 12, pp. 5369-5374, 2010.

[16] S. J. Remington, "Fluorescent proteins: maturation, photochemistry and photophysics," Current Opinion in Structural Biology, vol. 16, no. 6, pp. 714-721, 2006.

[17] J. D. Pédelacq, S. Cabantous, T. Tran, T. C. Terwilliger, and G. S. Waldo, "Engineering and characterization of a superfolder green fluorescent protein," Nature Biotechnology, vol. 24, no. 1, pp. 79-88, 2006.

[18] V. V. Verkhusha, H. Otsuna, T. Awasaki, H. Oda, S. Tsukita, and K. Ito, "An enhanced mutant of red fluorescent protein DsRed for double labeling and developmental timer of neural fiber bundle formation," The Journal of Biological Chemistry, vol. 276, no. 32, pp. 29621-29624, 2001.

[19] K. K. Turoverov, A. G. Biktashev, A. V. Dorofeiuk, and I. M. Kuznetsova, "A complex of apparatus and programs for the measurement of spectral, polarization and kinetic characteristics of fluorescence in solution," Tsitologiia, vol. 40, no. 8-9, pp. 806-817, 1998.

[20] I. M. Kuznetsova, O. V. Stepanenko, O. V. Stepanenko et al., "The place of inactivated actin and its kinetic predecessor in actin folding - unfolding," Biochemistry, vol. 41, no. 44, pp. 13127-13132, 2002.

[21] O. V. Stepanenko, V. V. Verkhusha, V. I. Kazakov et al., "Comparative studies on the structure and stability of fluorescent proteins EGFP, zFP506, mRFP1, "dimer2", and DsRed1," Biochemistry, vol. 43, no. 47, pp. 14913-14923, 2004.

[22] O. V. Stepanenko, I. M. Kuznetsova, V. V. Verkhusha, M. Staiano, S. D'Auria, and K. K. Turoverov, "Denaturation of proteins with beta-barrel topology induced by guanidine hydrochloride," Spectroscopy, vol. 24, no. 3-4, pp. 367-373, 2010.

[23] B. T. Andrews, A. R. Schoenfish, M. Roy, G. Waldo, and P. A. Jennings, "The rough energy landscape of superfolder GFP is linked to the chromophore," Journal of Molecular Biology, vol. 373, no. 2, pp. 476-490, 2007.

[24] B. T. Andrews, S. Gosavi, J. M. Finke, J. N. Onuchic, and P. A. Jennings, "The dual-basin landscape in GFP folding," Proceedings of the National Academy of Sciences of the United States of America, vol. 105, no. 34, pp. 12283-12288, 2008.

[25] B. T. Andrews, M. Roy, and P. A. Jennings, "Chromophore packing leads to hysteresis in GFP," Journal of Molecular Biology, vol. 392, no. 1, pp. 218-227, 2009. 


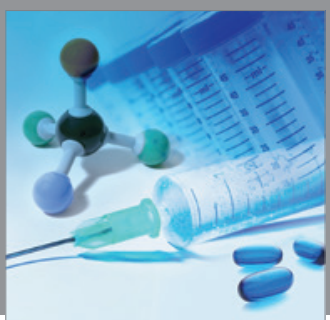

International Journal of

Medicinal Chemistry

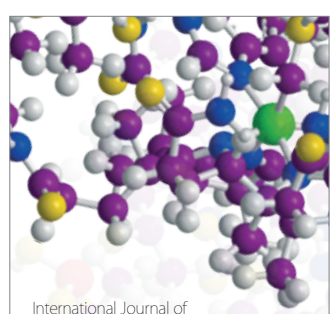

Carbohydrate Chemistry

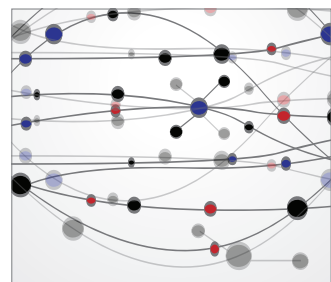

The Scientific World Journal
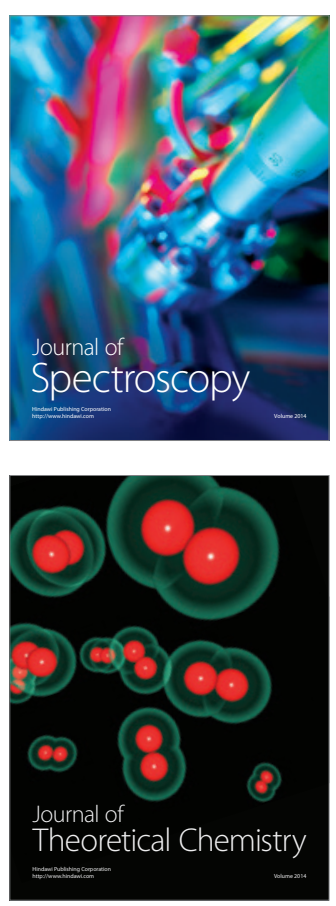
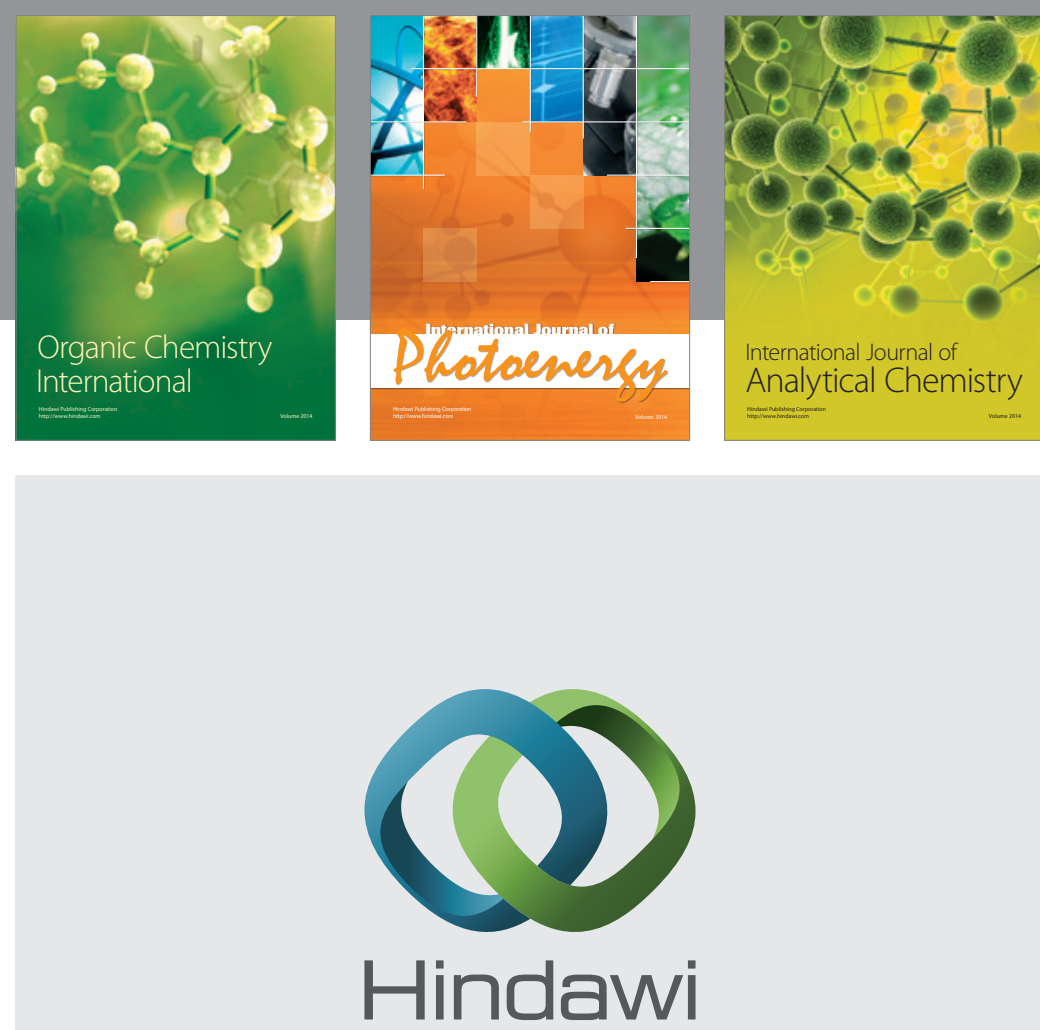

Submit your manuscripts at

http://www.hindawi.com
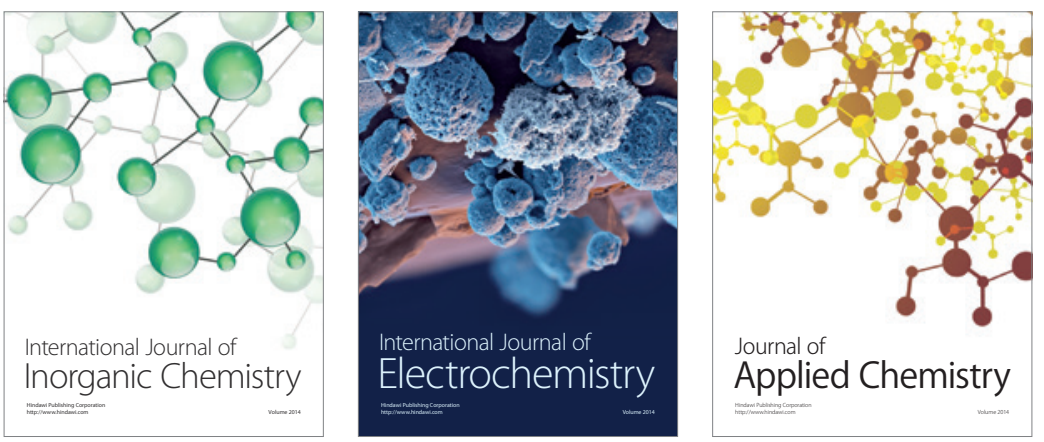

Journal of

Applied Chemistry
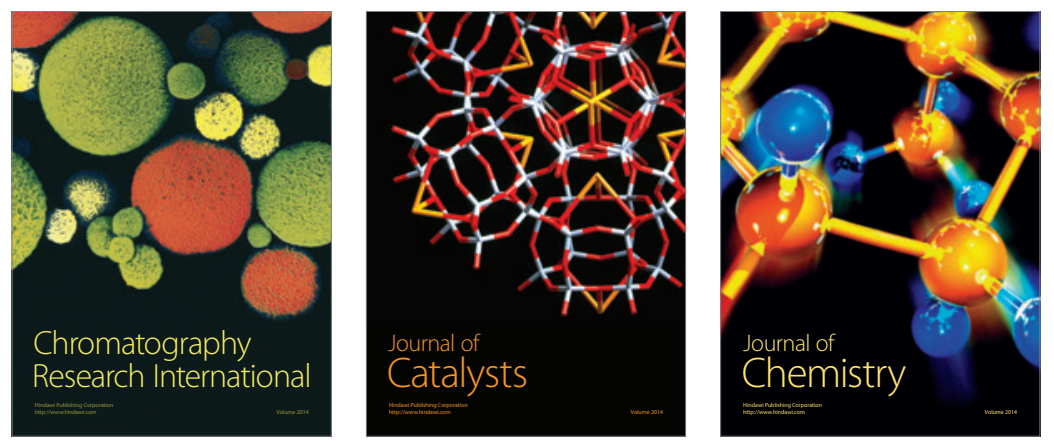
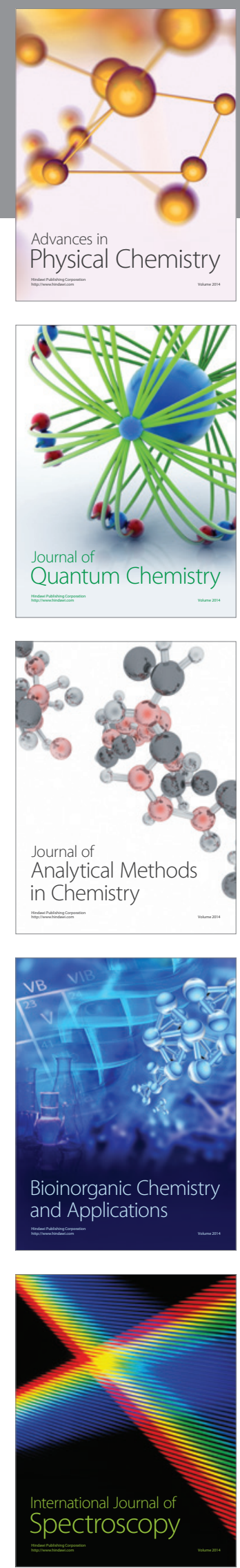